\title{
Karaya gum electrocardiographic electrodes for preterm infants
}

\author{
P H T CARTLIDGE AND N RUTTER
}

Department of Neonatal Medicine and Surgery, City Hospital, Nottingham

SUMMARY Changes in transepidermal water loss were used to measure skin damage caused by removal of electrocardiograph electrodes in 20 preterm infants. Electrodes secured by conventional adhesive damaged the skin, leading to a potentially dangerous increase in skin permeability. In contrast, those secured by karaya gum caused no skin damage.

The preterm infant's skin is seen as a convenient site for the application of monitoring probes during intensive care. When the probes are removed, however, the adhesive strips off the superficial layers of the stratum corneum causing discomfort to the infant and weakening the epidermal barrier ${ }^{1-3}$; electrodes used for electrocardiography are the biggest culprits. Electrocardiographic monitoring of the preterm infant is almost universal, the electrodes are much larger than other surface monitoring devices and they are the most difficult to remove. A new karaya gum electrode has been introduced for neonatal use, and early experience suggested that it caused less epidermal stripping than an electrocardiograph electrode with conventional adhesive. This study was devised to compare the skin damage caused by the two types of electrode.

\section{Subjects and methods}

Twenty preterm infants were studied. Their gestation ranged from 24 to 32 weeks (mean 29.7), birth weight 680 to $3020 \mathrm{~g}$ (mean 1500), and age from 1 to 10 days. An electrode with conventional adhesive (40426A Hewlett-Packard, United States) and one with karaya gum as the adhesive (Arbo-Pink H82V, West Germany) were fixed to opposite sides of the upper chest on undamaged skin. After 24 hours the electrodes were removed and skin damage assessed by measurement of transepidermal water loss with an evaporimeter (Ep 1, Servomed, Sweden) as previously described. ${ }^{4}$ The water loss was measured at the electrode site and at an adjacent untouched area of skin 10 minutes, four hours, 24 hours and, where possible, 48 hours after removal. Reaction of the infant to removal of the electrode and appearance of the skin were recorded. The study was approved by the hospital ethical committee and informed parental consent was obtained. Statistical analysis was by Wilcoxon's matched pairs signed rank test.

\section{Results}

Transepidermal water loss from the site of the conventional electrode was significantly higher than from adjacent skin with a mean of $83 \%$ higher at 10 minutes after removal and $32 \%$ higher at 48 hours (figure). By contrast, water loss from the site of the karaya gum electrode was not significantly different. When transepidermal water losses from the two electrode sites were compared, values from the conventional electrode site were higher than from the karaya gum electrode site on 63 of the 66 occasions that paired measurements were made. Removal of the conventional electrode was difficult and always painful and left an erythematous mark that lasted for several hours. The karaya gum electrode was easily and painlessly removed, and left no mark.

Both types of electrode gave good electrocardiographic traces. In two infants the karaya gum electrode fell off a total of four times (none of the conventional electrodes fell off), and on each occasion high ambient relative humidity $(85 \%)$ was being used. In vitro studies showed that the karaya gum tended to soften when the relative humidity exceeded $70 \%$ and started to disintegrate above $90 \%$.

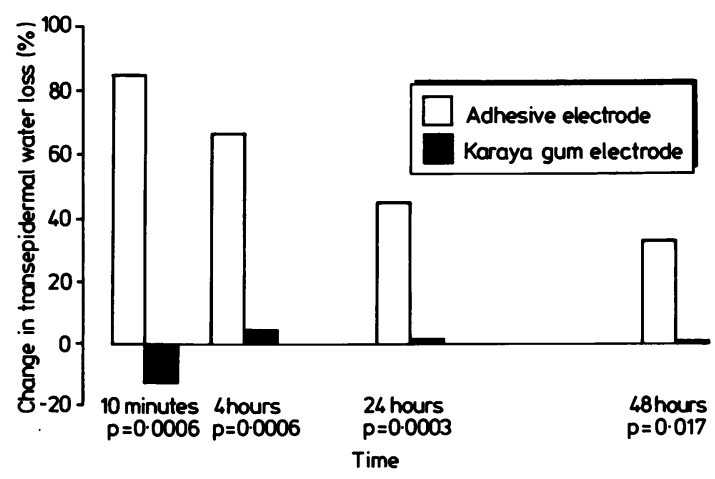

Figure Transepidermal water loss from the site of removal of electrocardiogram electrodes. 


\section{Discussion}

The marked and persistent rise in transepidermal water loss from the site of the conventional adhesive electrode indicated substantial damage caused by stripping of the epidermis. ${ }^{1}$ This explains why removal of the electrode was difficult and painful. In contrast, karaya gum stuck to the skin but did not strip the epidermis when removed, a property which makes it useful as a means of fixing a bag to an abdominal stoma. The thin skin of preterm infants is particularly vulnerable to trauma, as it lacks a well defined keratinised stratum corneum. ${ }^{5}$ Its already poor barrier properties are considerably weakened when the outermost layers of the epidermis are stripped off, which result in higher skin water loss and an increased permeability to potentially toxic drugs and chemicals. ${ }^{1}$ In addition, the damaged skin may be a site of entry for bacteria and result in local or systemic infection. ${ }^{1}$ It has previously been shown that the skin under the adhesive ring of transcutaneous oxygen electrodes is protected by the prior application of a spray on plastic dressing (Op-site) ${ }^{6}$ A further reduction in skin damage would result if karaya gum electrodes replaced conventional adhesive electrodes for electrocardiographic monitoring in preterm infants. They are three quarters the size of the conventional electrode used in this study (area of adhesion $6.5 \mathrm{~cm}^{2} v 8.5$ $\mathrm{cm}^{2}$ ) and half the cost.

After removal the karaya gum electrode remains sticky and can easily be resited. This is a valuable property that allows for convenient repositioning of the electrode during procedures such as chest transillumination, chest radiography, and the insertion of chest drains. Monitoring of heart rate was satis- factory with both electrodes, and the only problem encountered was reduced adhesion of the karaya gum electrode at high humidity. This was a minor inconvenience that led to a loss of the electrocardiographic trace on only four occasions in 480 hours of monitoring.

We conclude that conventional adhesive electrodes damage the preterm infant's skin and recommend that the more convenient karaya gum electrode is used instead for electrocardiographic monitoring: babies prefer them.

We are grateful to Dr DA Curnock and Professor AD Milner for allowing us to study infants under their care. We are indebted to the neonatal unit's nursing staff for their help and cooperation. PHTC was supported by a grant from the Medical Research Council of Great Britain.

\section{References}

1 Harpin VA, Rutter N. Barrier properties of the newborn infant's skin. $J$ Pediatr 1983:102:419-25.

${ }^{2}$ Pinkus M. Examination of the epidermis by the strip method of removing horny layers. 1 . Observations on thickness of the horny layer and on mitotic activity after stripping. $J$ Invest Dermatol 1951:16:383-6.

${ }^{3}$ Blank IH. Further observations on factors which influence the water content of the stratum corneum. J Invest Dermatol 1953 21: $259-69$.

${ }^{4}$ Nilsson GE. Measurement of water exchange through the skin Med Biol Eng Comput 1977:15:209-18.

5 Evans NJ, Rutter N. Development of the epidermis in the newborn. Biol Neonate 1986:49:74-80.

${ }^{6}$ Evans NJ, Rutter N. Reduction of skin damage from transcutaneous oxygen electrodes using a spray on dressing. Arch Dis Child 1986;61:881-4.

Correspondence to: Dr PHT Cartlidge, Department of Neonatal Medicine and Surgery. City Hospital, Nottingham NG5 1PB.

Received 24 June 1987 